\title{
Method for determination of resists parameters for photonic - integrated circuits e-beam lithography on silicon nitride platform
}

\author{
Ilia Elmanov$^{1}$, Anna Elmanova ${ }^{1}$, Sophia Komrakova ${ }^{1}$, Alexander Golikov ${ }^{1}$, \\ Natalya Kaurova ${ }^{1}$, Vadim Kovalyuk ${ }^{1,2}$, and Gregory Goltsman ${ }^{1,2,3}$ \\ ${ }^{1}$ Moscow State Pedagogical University, 119991 Moscow, Russia \\ ${ }^{2}$ Zavoisky Physical-Technical Institute of the Russian Academy of Sciences, 420029 Kazan, Russia \\ ${ }^{3}$ National Research University Higher School of Economics, 101000 Moscow, Russia
}

\begin{abstract}
In the work the thicknesses of the e-beam resists ZEP 520A and ma-N 2400 by using non-destructive method were measured, as well as recipe for the high ratio between the $\mathrm{Si}_{3} \mathrm{~N}_{4}$ and the resists etching rate was determined. The work has a practical application for e-beam lithography of photonic-integrated circuits and nanophotonics devices based on silicon nitride platform.
\end{abstract}

Electron-beam lithography is one of the most important instruments for development of photonic-integrated circuits and nanophotonics devices of several nanometers' scales, where high quality e-beam resists play a key role for images and protect nanostructures during etching processes [1]. There are many different electronic resists with different performance. By the way of getting images they can be divided on two types: positive (exposed areas are removed after development and mask is formed from the resting resist) and negative ones (defending mask is formed in the exposure radiated areas and the rest part is removed during development).
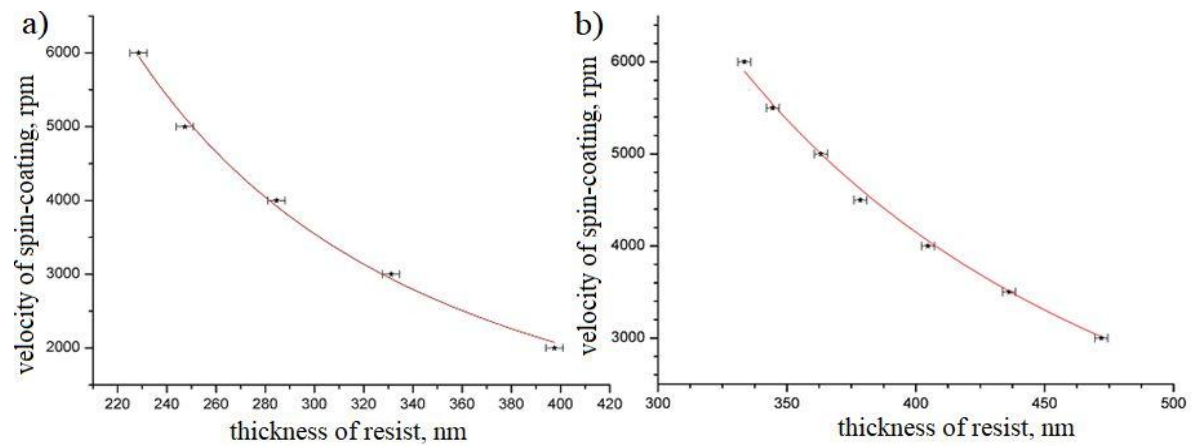

Fig. 1. Experimentally obtained thicknesses of the resists ma-N 2403 (a) and ZEP 520A (b) spin-coater rotation speed. The second order power function was used. 

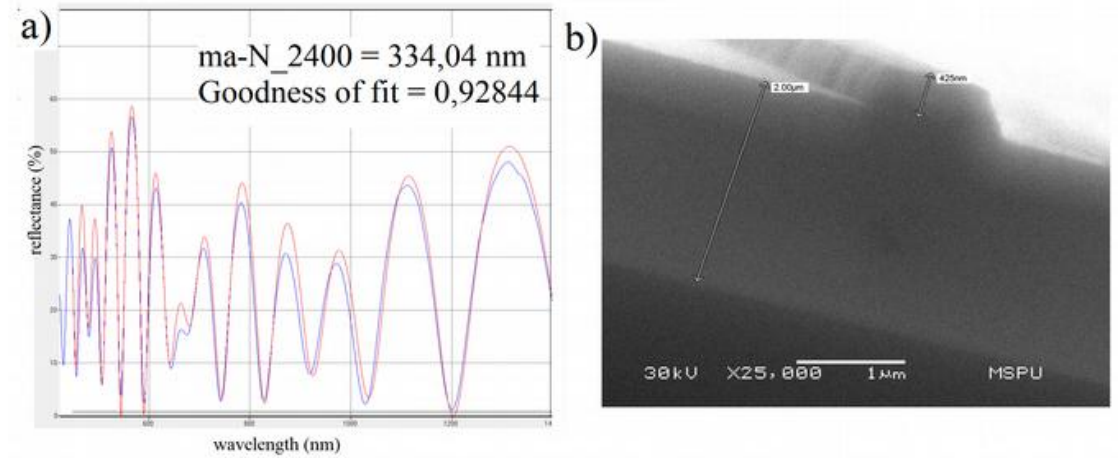

Fig. 2. Thickness of resist ma-N 2403 before etching, measured using Filmetrix F20 (a) and the waveguide cross-section with ma-N 2403 atop after etching (b).

In practice, it is better to use as minimal layer of the resist as it is possible in order to minimize proximity effects on the forming structures and get smaller sizes of separated elements. In the same time, while etching the structures the resist is also etched. Therefore, it is necessary to identify the conditions. where the etching rate of $\mathrm{Si}_{3} \mathrm{~N}_{4}$ in comparison with resist etching rate will be much higher. To do that, the thicknesses of the resists should be measured and the $\mathrm{Si}_{3} \mathrm{~N}_{4}$ etching rate should be studied, as well as the recipe parameters should be determined.

In this work we studied two widely used electronic resists: ZEP 520A (positive) [2] and ma-N 2400 (negative) [3]. After resists spin-coating the thicknesses of the resists by non-destroying method base on reflectometer (Filmetrix F20) were measured. The error bars, depicted in Fig.1, where calculated after series of thickness measurements of the resits. The error value was less than $4 \mathrm{~nm}$ that is a high precision of the results.

The recipe was obtained for RIE using parameters: work pressure - 55 mTorr, flux of $\mathrm{CHF}_{3}$ $-50 \mathrm{sccm}, \mathrm{O}_{2}-2 \mathrm{sccm}$, providing the ratio of the etching of resist to the rate of etching of $\mathrm{Si}_{3} \mathrm{~N}_{4}$ equal 0.44 and 0.51 for ma-N 2400 and ZEP 520A, respectively.

In the Fig. 2 microphotograph of $\mathrm{Si}_{3} \mathrm{~N}_{4}$ waveguide cross-section with developed resist atop is shown. In the Fig.2 (a) the reflectance spectra from the multilayer sample (ma-N $2400, \mathrm{Si}_{3} \mathrm{~N}_{4} 200 \mathrm{~nm}, \mathrm{SiO}_{2} 2 \mu \mathrm{m}$ and silica substrate) is shown. The obtained results allow us to choose the better resists parameters for the application due to the type of structures and the needed width of etching. For the mentioned before recipe for etching of $200 \mathrm{~nm} \mathrm{Si} \mathrm{N}_{4}$ the minimal width of resist should be $90 \mathrm{~nm}$ for ma-N 2400 and $105 \mathrm{~nm}$ for ZEP 520 A.

The work supported by Ministry of Education and Science of the Russian Federation (agreement №14.583.21.0065, unique identificatory: RFMEFI58317X0065).

\section{References}

1. Wayne M. Moreau, Semiconductor Lithography. Principles, Practices, and Materials. Plenum Press, 1988 ISBN 0-306-42185-2

2. ZEP 520A. Technical Report. Ver. 1.01. ZEONREX Electronic Chemicals, 2003.

3. Ma-N 2400 - Negative Tone Photoresist Series. Micro resist technology GmbH, 2012. 